\title{
Measurement and analysis of excitation functions and observation of mass-asymmetry effect on incomplete fusion dynamics
}

\author{
D. Singh ${ }^{1, a}$, Rahbar Ali², M. Afzal Ansari ${ }^{2}$, M. H. Rashid ${ }^{3}$, and R. Guin ${ }^{3}$ \\ 1 Nuclear Physics Division, Inter-University Accelerator Centre, New Delhi-110067, India, \\ 2 Department of Physics, Aligarh Muslim University, Aligarh-202002, India, \\ 3 Variable Energy Variable Energy Cyclotron Centre, 1/AF, Bidhan Nagar, Kolkata-700064, India
}

\begin{abstract}
Excitation functions for sixteen evaporation residues produced in the interaction of ${ }^{20} \mathrm{Ne}$ with ${ }^{165} \mathrm{Ho}$ have been measured in the projectile energy range $\approx 88-164 \mathrm{MeV}$, using catcher foil activation technique followed by gamma-ray spectrometry. It has been found in general that the excitation functions of evaporation residues produced via $\mathrm{xn} / \mathrm{pxn}$ channels satisfactorily reproduced with the statistical model code PACE-2 after subtraction of precursor decay contribution. The significant enhancement in the measured excitation functions for the residues produced in alpha emission channels over the PACE-2 predictions has been observed. These alpha emission channels are attributed to incomplete fusion reaction process. The results indicate the occurrence of incomplete fusion involving break-up of projectile ${ }^{20} \mathrm{Ne}$ into ${ }^{4} \mathrm{He}+{ }^{16} \mathrm{O}$ and $/$ or ${ }^{8} \mathrm{Be}+{ }^{12} \mathrm{C}$ followed by fusion of one of the fragments with target nucleus ${ }^{165} \mathrm{Ho}$. The analysis of the present data suggest that probability of incomplete fusion increases with projectile energy. The ICF fraction $F^{I C F}$ also increases with increasing mass-asymmetry of the entrance channel.
\end{abstract}

\section{Introduction}

The study of incomplete fusion (ICF) dynamics in heavy ion (HI) reactions particularly with heavier target nuclei above Coulomb barrier has been the subject of growing interest in nuclear physics from past few decades. It has been observed that at projectile energies slightly above the Coulomb barrier, both the complete fusion (CF) and incomplete fusion (ICF) may be considered as dominant reaction mechanisms. In case of $\mathrm{CF}$ reaction the projectile completely fuses with the target nucleus and the highly excited nuclear system decays by evaporating low energy nucleons and alpha particles, which is well explained by statistical model. In the ICF reaction process, only a part of the projectile fuses with target nucleus, while remaining part moves in the forward direction [1-3]. The excited composite system formed as a result of the fusion of the fragment of the incident ion with the target may also under go de-excitation by emission of particles and/or $\gamma$-rays. The first experimental evidence of ICF reactions was given by Britt et al. [4], who observed the break-up of the incident projectiles like ${ }^{12} \mathrm{C},{ }^{14} \mathrm{~N}$ and ${ }^{16} \mathrm{O}$ into alpha clusters in an interaction with the surface of the target nucleus at $\approx 10.5 \mathrm{MeV} /$ nucleon bombarding energies. The major advances in the understanding of ICF dynamics were made after the charged particle- $\gamma$ coincidence measurements by Inamura et al. [5]. The studies by Morgestern et al. [6] carried out experiments on the various projectile target combinations, have brought out the entrance channel mass-asymmetry dependence of ICF reaction, with ICF probability being higher in a mass-asymmetric system than mass-symmetric system at same relative velocity. Later on, studies by Vineyard et al. [7] and Chakrabarty et al. [8], Ali et al. [9] and

\footnotetext{
a e-mail: dsinghiuac@gmail.com
}

Singh et al. [10] also supported the findings of Morgestern et al. [6]. However, their studies are limited to few projectiletarget combinations. Systematic measurements are however still demanded. Several dyanamical models [11-13] have been proposed to understand ICF dynamics but none of those is able to fit the experimental data at relatively low bombarding energies (i.e. 4-8 MeV/nucleon). Hence, the study of ICF dynamics is still an active area of investigation. Most of the ICF studies have been carried out with low Z-projectile $(\mathrm{Z} \leq 10)$, induced reactions on targets of medium mass $(\mathrm{A}<120)$. However, such information is scarce for heavier-mass target nuclei. In the present work the excitation functions (EFs) of sixteen evaporation residues (ERs) produced via complete and incomplete fusion in the reaction of ${ }^{20} \mathrm{Ne}$ with ${ }^{165} \mathrm{Ho}$ have been measured in the energy range $\approx 4-8 \mathrm{MeV} /$ nucleon. In the present measurement special care has been taken to remove the precursor decay contributions in the production of several ERs to get the direct production cross-sections of the residues. The measured EFs have been compared with the statistical model code PACE-2 [14], which takes into account only the CF process and the enhancement in the measured EFs are interpreted in terms of ICF process. The RRDs of the present projectile-target system have been measured at $\approx 8$ $\mathrm{MeV} /$ nucleon projectile energy and are recently published and reported elsewhere in Ref. [15]. In the present work the dependence of ICF fraction with projectile energy has also been discussed. The entrance channel mass-asymmetry dependence on ICF fraction are also investigated and plotted along with similar data on different projectile-target systems ${ }^{20} \mathrm{Ne}+{ }^{59} \mathrm{Co}[10],{ }^{16} \mathrm{O}+{ }^{45} \mathrm{Sc}[16]$ and ${ }^{16} \mathrm{O}+{ }^{74} \mathrm{Ge}$ [16]. The present work is a part of ongoing program $[9,10$, $15,17-19]$ to study the CF and ICF dynamics in heavy ion induced reactions below $10 \mathrm{MeV} /$ nucleon energies. To the best of our knowledge these measurements for the projectile-

This is an Open Access article distributed under the terms of the Creative Commons Attribution-Noncommercial License 3.0, which permits unrestricted use, distribution, and reproduction in any noncommercial medium, provided the original work is properly cited. 
target system mentioned above have been reported for the first time.

\section{Experimental details}

The present experiment has been performed at Variable Energy Cyclotron Centre (VECC), Kolkata, India. Selfsupporting natural ${ }^{165} \mathrm{Ho}$ targets of desired thickness with purity better than $(99.9 \%)$ were prepared by rolling machine. The thickness of each target foils was determined using microbalance as well as by $\alpha$-particle transmission method. Two stacks consisting of six rolled holmium foils each of ${ }^{165} \mathrm{Ho}$ backed by thick aluminum foils were bombarded with the ${ }^{20} \mathrm{Ne}^{+7}$ ion beam of energy $\approx 165$ and 132 $\mathrm{MeV}$ in a specially designed vacuum chamber. Keeping in view the half-lives of interest, irradiations have been carried out for $\approx 8$ hours duration for each stack. Moreover, when beam passes through the target and catcher foils, the energy spread due to straggling may come into picture. However, the energy spread due to straggling has not been considered due to its non-significant contribution [20]. The mean energy of ${ }^{20} \mathrm{Ne}$-ion beam incident at half the thickness on each foil in the stack was calculated from the energy degradation of the incident beam energy, using software SRIM [21]. The gamma-ray activities produced in various targets and successive catcher foils were then identified by counting them on a HPGe detector, coupled to a PC based data acquisition system. Softwares MAESTRO [22] and FREEDOM [23] have been used for recording and analysis of the data respectively. The energy calibration of the detector was done by using ${ }^{152} \mathrm{Eu} \gamma$-ray source of known strength.The ERs were identified by their characteristic $\gamma$-rays as well as following their half-lives. The characteristic $\gamma$-rays energies, half-lives of residual nuclei, branching ratio of the $\gamma$-rays, abundance, etc. are taken from the Table of Isotopes [24]. Some evaporation residues are found to have contribution from precursor decay, which has been separated out from the measured cumulative crosssection of evaporation residues using prescription given in Ref. [10]. The experimentally measured reaction crosssection, for a particular reaction product has been computed using the expression given in Ref. [10]. The details of error and uncertainties in the experimentally measured data have been given in our earlier Ref. [9].

\section{Analysis of the experimental data using statistical Model code PACE-2}

The theoretical estimates of the reaction cross-sections were made by the statistical model code PACE-2 [14]. In this code the most of required input parameters have been used as default except the charge and mass of the projectile and target nucleus. The excitation functions for sixteen evaporation residues ${ }^{182} \operatorname{Ir}(3 \mathrm{n}),{ }^{182} \mathrm{Os}(\mathrm{p} 2 \mathrm{n}),{ }^{181} \mathrm{Os}(\mathrm{p} 3 \mathrm{n}),{ }^{181} \operatorname{Re}(\alpha)$, ${ }^{179} \operatorname{Re}(\alpha 2 \mathrm{n}), \quad{ }^{178} \operatorname{Re}(\alpha 3 \mathrm{n}), \quad{ }^{177} \operatorname{Re}(\alpha 4 \mathrm{n}), \quad{ }^{177} \mathrm{~W}(\alpha \mathrm{p} 3 \mathrm{n})$, ${ }^{174} \mathrm{~W}(\alpha \mathrm{p} 6 \mathrm{n}), \quad{ }^{178 m} \mathrm{Ta}(\alpha 2 \mathrm{pn}), \quad{ }^{177} \mathrm{Ta}(2 \alpha), \quad{ }^{176} \mathrm{Ta}(2 \alpha \mathrm{n})$, ${ }^{175} \mathrm{Ta}(2 \alpha 2 \mathrm{n}),{ }^{174} \mathrm{Ta}(2 \alpha 3 \mathrm{n}),{ }^{173} \mathrm{Hf}(2 \alpha \mathrm{p} 3 \mathrm{n})$ and ${ }^{166} \mathrm{Tm}(4 \alpha 3 \mathrm{n})$ produced in the interaction of ${ }^{20} \mathrm{Ne}$ with ${ }^{165} \mathrm{Ho}$ have been measured. The EF for evaporation residue ${ }^{182} \mathrm{Ir}$ is measured directly and is shown in Fig. 1(a). The effect of variation of ' $\mathrm{K}$ ' $(=10,12,14)$ on the calculated EFs for the evaporation residues produced in the reactions ${ }^{182} \operatorname{Ir}(3 \mathrm{n})$ and ${ }^{182} \mathrm{Os}(\mathrm{p} 2 \mathrm{n})$ are shown in Figs. 1(a)-(b). It is quite clear from these figures that PACE-2 predictions corresponding to level density parameter constant $\mathrm{K}=12$ reproduce the measured EFs satisfactorily and these reaction channels are populated via complete fusion process whereas, the projectile ${ }^{20} \mathrm{Ne}$ fuses with target ${ }^{165} \mathrm{Ho}$ and the compound system ${ }^{185}$ Ir decays by emission of $3 \mathrm{n}$ and $\mathrm{p} 2 \mathrm{n}$. In the similar way, the measured direct cross-sections for the evaporation residue ${ }^{181} \mathrm{Os}(\mathrm{p} 3 \mathrm{n})$ has been compared with PACE-2 predictions corresponding to level density parameter constant $\mathrm{K}=12$ reproduce the measured $\mathrm{EF}$ satisfactorily. It means that the $\mathrm{ER}^{181} \mathrm{Os}(\mathrm{p} 3 \mathrm{n})$ is also populated through CF only. The measured direct cross-sections for the production of evaporation residue ${ }^{181}$ Re compared with PACE-2 calculated values, as shown in the Figs. 1(c), it is observed that the measured direct EFs are much enhanced over their theoretical values. Since ICF is not considered in PACE2 calculations, this enhancement may be attributed to the fact that this channel may be populated not only by CF of ${ }^{20} \mathrm{Ne}$ but may also have a significant contribution from ICF of ${ }^{20} \mathrm{Ne}$. Similarly, the measured direct cross-sections for the ERs ${ }^{179} \mathrm{Re},{ }^{178} \mathrm{Re},{ }^{177} \mathrm{Re},{ }^{177} \mathrm{~W},{ }^{177} \mathrm{Ta},{ }^{176} \mathrm{Ta},{ }^{175} \mathrm{Ta}$, ${ }^{174} \mathrm{Ta},{ }^{178 m} \mathrm{Ta}$ and ${ }^{173} \mathrm{Hf}$ also compared with PACE-2 predictions. The measured direct cross-sections are comparatively much larger than PACE-2 predictions. This large enhancement in the measured cross-sections than their theoretical predictions may be attributed to the ICF process. It means that these ERs may be populated not only by $\mathrm{CF}$ of ${ }^{20} \mathrm{Ne}$ but may also be populated through ICF of ${ }^{20} \mathrm{Ne}$. In case of evaporation residue ${ }^{166} \mathrm{Tm}$ produced via $4 \alpha 3 \mathrm{n}$ emissions channel the theoretical prediction of code PACE-2 gives negligible cross-sections $(<0.01 \mathrm{mb})$, and hence are not shown in the Fig.1(d), while the measured crosssections are comparatively much larger. This large enhancement in the measured cross-sections than their theoretical predictions may be attributed to the ICF process of the projectile ${ }^{20} \mathrm{Ne}$ (if ${ }^{20} \mathrm{Ne}$ breakup into ${ }^{4} \mathrm{He}$ and ${ }^{16} \mathrm{O}$ fragments and fusion of ${ }^{4} \mathrm{He}$ fragment with target ${ }^{165} \mathrm{Ho}$ and emission of 3 neutrons takes place from the composite system). Another possibility of ICF process may be understood by the breakup of the projectile ${ }^{20} \mathrm{Ne}$ into ${ }^{8} \mathrm{Be}$ and ${ }^{12} \mathrm{C}$ and fusion of fragment ${ }^{8} \mathrm{Be}$ with the target and emission of $1 \alpha$-particle and 3 neutrons from the composite system.

\section{ICF fraction and mass-asymmetry effect}

The enhancement in the experimentally measured production cross-sections over the PACE- 2 predictions in some of the residues may be attributed to ICF process. As such the ICF contribution for individual channels has been estimated by subtracting theoretically calculated CF crosssection by PACE- 2 from the experimentally measured crosssections at each projectile energy. The detail discussion regarding the calculation of total ICF cross-section $\left(\sum \sigma^{I C F}\right)$ , total fusion cross-section $\left(\sum \sigma^{T F}\right)$, total CF cross-section $\left(\sum \sigma^{C F}\right)$, and ICF fraction $\left(F^{I C F}\right)$ are given in Ref. [10]. The total CF channels $\left(\sum \sigma^{C F}\right)$ and total ICF channels $\left(\sum \sigma^{I C F}\right)$ contribution along with total fusion reaction cross-section $\left(\sum \sigma^{T F}\right)$ for the ${ }^{20} \mathrm{Ne}+{ }^{165} \mathrm{Ho}$ system is plotted against projectile energy as shown in Fig. 2.(a). It has been observed that ICF contributes larger production yield with respect to the CF process as the projectile energy is 

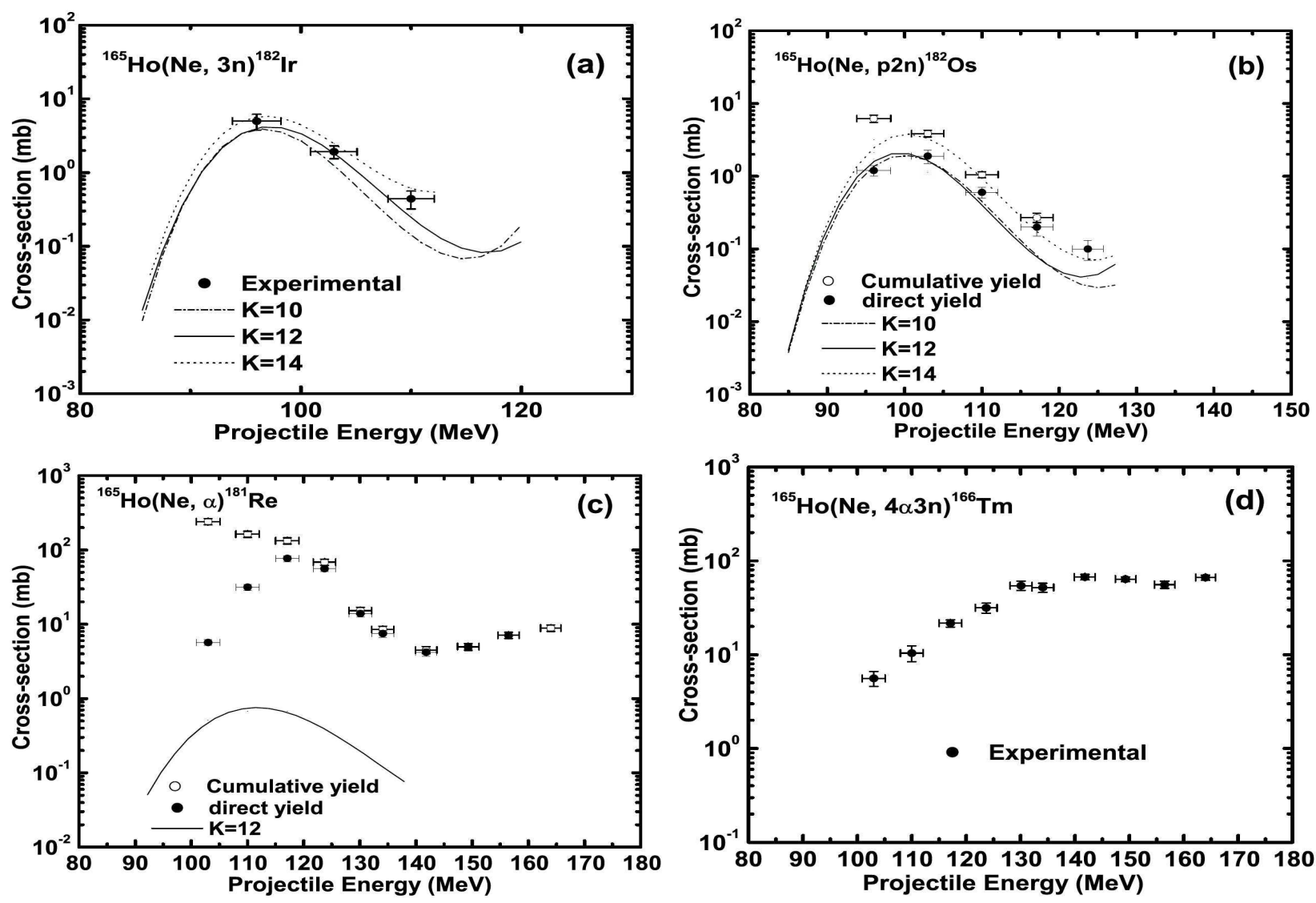

Fig. 1. Excitation function of the evaporation residues in ${ }^{20} \mathrm{Ne}+{ }^{165} \mathrm{Ho}$ reaction. Circles represent experimental data. The solid, dotted and dash dotted lines correspond to the theoretical predictions of PACE-2 for different values of level density parameter constant K.

increased, which is expected as the break-up probability of the incident ion into $\alpha$-clusters increases with projectile energy. The incomplete fusion fraction, $F^{I C F}$ has been plotted as a function of projectile energy for the system ${ }^{20} \mathrm{Ne}+{ }^{165} \mathrm{Ho}$ as shown in Fig. 2(b). It is observed from the figure that ICF fraction invariably increases with projectile energy for ${ }^{20} \mathrm{Ne}+{ }^{165} \mathrm{Ho}$ system. Morgenstern et al. [6] has suggested that onset of incomplete fusion is governed by relative velocity of projectile $V_{\text {rel }}$, given by:

$$
V_{\text {rel }}=\sqrt{\frac{2\left(E_{C M}-E_{C B}\right)}{\mu}}
$$

where, $\mu$ is the reduced mass of the system, $E_{C M}$ is the centre-of-mass energy and $E_{C B}$ is the Coulomb barrier between two interacting partners. This expression takes into account the difference in Coulomb barrier between each two systems. Keeping in view, the percentage of incomplete fusion fraction $F^{I C F}$ has been deduced for the same $V_{\text {rel }}(0.081 \mathrm{c})$ and plotted as a function of mass-asymmetry between projectile and target as shown in Fig. 2(c). The figure shows that the ICF fraction $F^{I C F}$ is sensitive to projectile energy and mass-asymmetry of the projectile-target system and in general ICF probability is more in a massasymmetric system than in mass-symmetric system.

\section{Summary and conclusion}

The excitation functions for sixteen evaporation residues produced in complete and/or incomplete fusion process in
${ }^{20} \mathrm{Ne}+{ }^{165} \mathrm{Ho}$ systems have been measured in beam energy range $\approx 88-164 \mathrm{MeV}$. Comparison of the measured excitation functions with statistical model code PACE-2 calculation values has been done. The enhancement in the measured cross-section (direct yield) for the evaporation residues produced in $\alpha$-emission channels $\operatorname{Viz} ;{ }^{181} \operatorname{Re}(\alpha)$, ${ }^{179} \operatorname{Re}(\alpha 2 \mathrm{n}), \quad{ }^{178} \operatorname{Re}(\alpha 3 \mathrm{n}), \quad{ }^{177} \operatorname{Re}(\alpha 4 \mathrm{n}), \quad{ }^{177} \mathrm{~W}(\alpha \mathrm{p} 3 \mathrm{n})$, ${ }^{174} \mathrm{~W}(\alpha \mathrm{p} 6 \mathrm{n}), \quad{ }^{178 m} \mathrm{Ta}(\alpha 2 \mathrm{pn}), \quad{ }^{177} \mathrm{Ta}(2 \alpha), \quad{ }^{176} \mathrm{Ta}(2 \alpha \mathrm{n})$, ${ }^{175} \mathrm{Ta}(2 \alpha 2 \mathrm{n}),{ }^{174} \mathrm{Ta}(2 \alpha 3 \mathrm{n}),{ }^{173} \mathrm{Hf}(2 \alpha \mathrm{p} 3 \mathrm{n})$ and ${ }^{166} \mathrm{Tm}(4 \alpha 3 \mathrm{n})$ over their theoretical predictions based on complete fusion model attributed to the fact that these residues are not only formed by the complete fusion but also through incomplete fusion process. Subsequent emission of neutrons and protons during the thermalization of the composite system leads to the production of above evaporation residues populated through ICF process. The analysis of the data also suggests that ICF probability increases with projectile energy. The comparison of the present data with similar data on systems ${ }^{20} \mathrm{Ne}+{ }^{59} \mathrm{Co}[10],{ }^{16} \mathrm{O}+{ }^{45} \mathrm{Sc}$ and ${ }^{16} \mathrm{O}+{ }^{74} \mathrm{Ge}[16]$ suggests that ICF probability increases in mass-asymmetric system than in mass-symmetric system.

\section{Acknowledgements}

The authors are grateful to the Variable Energy Cyclotron Centre, Kolkata, for providing the experimental facilities to carry out the experiment. The authors are also thankful to operational staff of cyclotron, VECC, Kolkata for good co-operation during the course of this experiment. Travel 

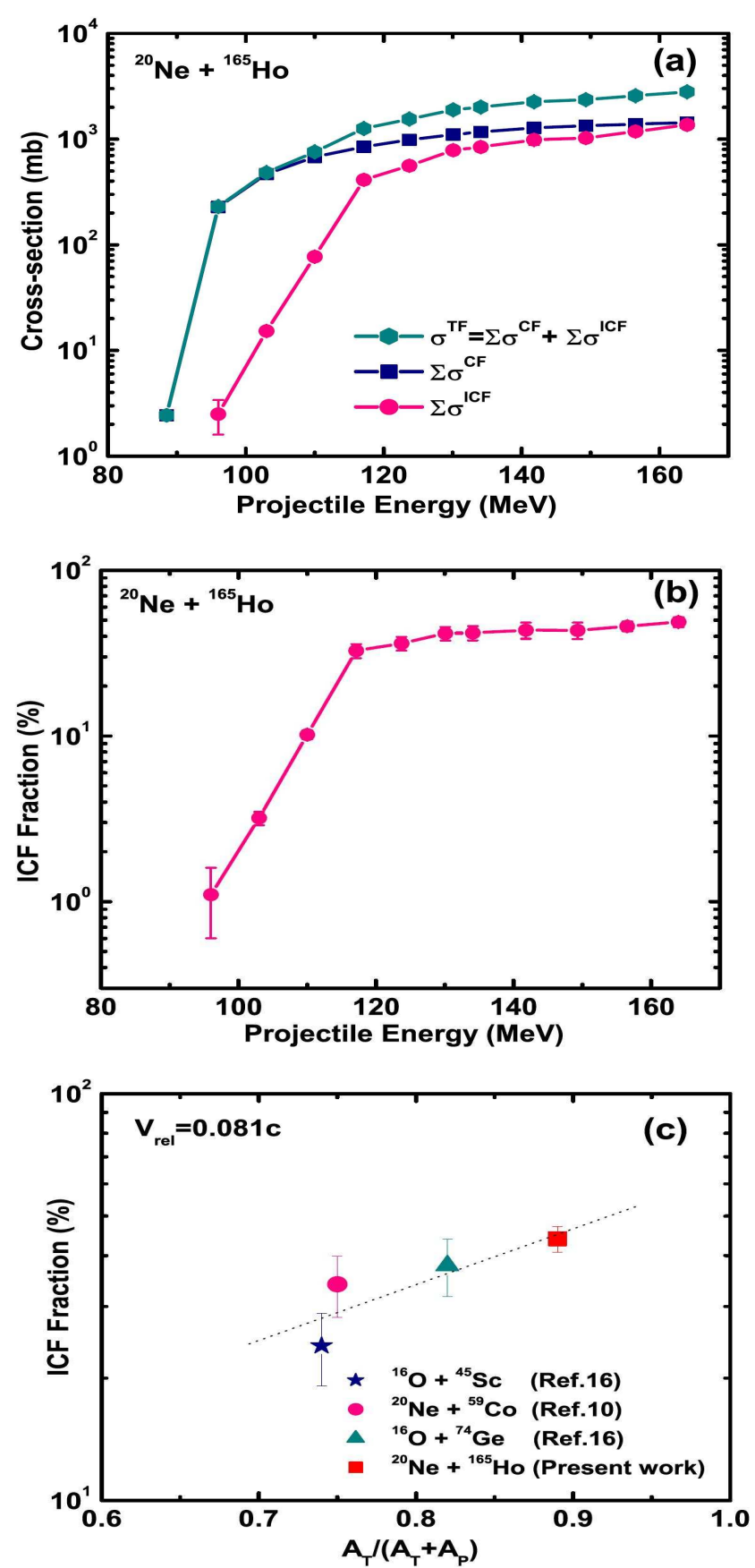

Fig. 2. (Colour online) Total fusion cross-section $\left(\sum \sigma^{T F}\right)$ along with the sum of CF cross-sections $\left(\sum \sigma^{C F}\right)$ and sum of ICF crosssections $\left(\sum \sigma^{I C F}\right)$ at different projectile energy for the system ${ }^{20} \mathrm{Ne}+{ }^{165} \mathrm{Ho}$. (b) ICF fraction $\left(F^{I C F}\right)$ as a function of projectile energy. (c) ICF fraction $\left(F^{I C F}\right)$ as a function of mass-assymmetry in different projectile-target systems.

\section{References}

1. P. E. Hodgson,Nuclear Heavy Ion Reactions (Clarendon Press, Oxford, 1978).

2. P. E. Hodgson et al., Introductory Nuclear Physics Clarendon Press. Oxford 1997).

3. M. Cavinato et al., Heavy Ion Fusion: Exploring the variety of nuclear properties (Editor, A. M. Stefanini et al., p.180, World Scientific, Singapore, 1994).

4. H. C. Britt et al., Phys. Rev. 124, (1964) 877.

5. T. Inamura et al., Phys. Lett. B68, (1977) 51 .

6. H. Morgenstern et al., Phys. Rev. Lett. 52, (1984) 1104.

7. M. F. Vineyard et al., Phys. Rev. C 45, (1992) 1784.

8. S. Chakrabarty et al., Nucl. Phys. A 678, (2000) 355 .

9. R. Ali et al., J. Phys. G: Nucl. Part. Phys. 37, (2010) 115101 .

10. D. Singh et al., Phys. Rev.C 83, (2011) 054604.

11. T. Udagawa et al., Phys. Rev. Lett. 45,(1980) 1311.

12. J. Wilczynski et al., Nucl. Phys. A 373,(1982) 109.

13. P. Bondrof et al., Nucl. Phys. A 333, (1980) 285 .

14. A. Gavron, Phys. Rev. C, 21, (1980) 230 .

15. D. Singh et al., Phys. Rev.C 79, (2009) 054601.

16. D. Singh, Ph. D. Thesis, Aligarh Muslim University, Aligarh, India (2008).

17. D. Singh et al., Jour. Phys. Soci. Japn. 75, (2006) 104201 .

18. D. Singh et al., Chin. J. Phys. 46, (2008) 27 .

19. D. Singh et al., Physical Review C 81, (2010) 027602.

20. B. Wilken et al., Fritz: Nucl. Instr. Meth. 138, (1976) 331 .

21. J. F. Ziegler, SRIM-2006, The Stopping Power and Range of Ions in Matter, 2006.

22. MAESTRO, Data acquisition and analysis software coupled with EG \& G ORTEC hardware.

23. FREEDOM, Data acquisition and analysis system designed to support the accelerator based experiments at the Inter University Accelerator Centre, New Delhi, India

24. R. B. Firestone et al., Table of Isotopes, 8th edition (Wiley, New York, 1996). support provided by UGC-DAE-CSR, Kolkata is acknowledged gratefully. The authors are also thankful to the Department of Physics, A.M.U, Aligarh, India, for providing the necessary facilities related with this research work. One of the author (D.S.) is also thankful to the Council of Scientific and Industrial Research (CSIR), New Delhi for providing the financial support during this research work. 